\title{
On the Peak-to-Average Power Ratio of Pre-Equalized Base-Field Hartley OFDM
}

\author{
Lin Luo*, Chung Shue Chen ${ }^{\dagger}$ and Siu-Wai $\mathrm{Ho}^{*}$ \\ *Institute for Telecommunications Research, University of South Australia, Australia \\ Email: \{Lin.Luo, Siuwai.Ho\}@unisa.edu.au \\ †Alcatel-Lucent Bell Labs, Centre de Villarceaux, 91620 Nozay, France \\ Email: cs.chen@alcatel-lucent.com
}

\begin{abstract}
We show by the analytical and simulation results that pre-equalized OFDM based on base-field Hartley transform (BHT-OFDM), which is an alternative air interface for LTEbeyond or 5G, provides significantly better PAPR performance than conventional OFDM systems. We develop a tractable analytical closed-form expression of the statistical distribution of PAPR of pre-equalized BHT-OFDM based on correlated multivariate Rayleigh distributions and verify the result with comprehensive simulation studies. Comparisons show that the analytical and simulation results are consistent. Our study suggests that preequalized BHT-OFDM is not only favorable due to its inherent error correction capability but is also highly recommendable for its superiority in power efficiency beyond today's OFDM scheme.
\end{abstract}

\section{INTRODUCTION}

OFDM is widely deployed in todays wireless communication systems including IEEE 802.11, WiMax and 3GPP Long Term Evolution (LTE) and will be used by emerging LTEAdvanced (LTE-A) [1]. However, a major drawback of OFDM transmission is that its signal waveform usually has a high peak-to-average power ratio (PAPR) and hence it generally yields heavy power penalty and reduces the energy utilization efficiency at transmitters. Although there are techniques to tackle this problem and alleviate the inherent difficulties, existing work and results mainly focus on patching the conventional OFDM schemes [2]. Recently a pre-equalized OFDM based on basefield Hartley transform (BHT-OFDM) has been proposed [3]. Unlike conventional pre-equalization technique for OFDM, which only shifts the equalization from the receiver to the transmitter and suffers even worse PAPR, the pre-equalized BHT-OFDM operates over a finite field. Therefore the dynamic range of signal at BHT-OFDM transmitter is naturally confined to the range of a finite alphabet set, which would significantly narrow the range of the signal energy.

Notice that existing theoretical upper bound of PAPR (e.g., the PAPR in OFDM signals with $N$ subcarriers and phase-shift keying (PSK) modulation is upper bounded by $N$ ) may not be very meaningful for characterizing the performance of actual power consumption in the OFDM signals. The statistical distribution of PAPR has to be taken into account in practice. They are classified into empirical and theoretical expressions [4], [5], [6]. Generally speaking, the existing empirical analysis [4] lacks a very rigorous theoretical justification although it may work in some cases, and the existing theoretical expressions have high computational complexity with several difficult integrals, see [5], [6]. The conventional analysis of the PAPR of OFDM signals will be overviewed in Section III.

Besides, most of existing analyses claimed that their empirical or theoretical results are suitable for large subcarrier number $N$. This is usually for mathematical approximation and simplicity, see e.g. [6]. Explicit closed-form expression and resulting performance guidelines for all $N$ would be highly favorable to the future use of OFDM and its various in LTE/LTE-A/4G/beyond $4 \mathrm{G}$ mobile radio systems and for energy-efficient wireless communications, especially to preequalized BHT-OFDM systems.

The contributions of this paper are summarized as follows:

- Develop a tractable analytical closed-form expression of PAPR of BHT-OFDM based on correlated multivariate Rayleigh distributions. The accurate analysis is suitable for other communication systems with correlated signals.

- Theoretically analyse the high energy efficiency of BHTOFDM, and justify its validation by simulation over several $4 \mathrm{G}$ channel models. BHT-OFDM is approved to be a highly considerable candidate beyond OFDM for next generation communication systems.

The rest of the paper is organized as follows. Section II describes the system model and problem setup. Section III presents existing conventional analysis of the PAPR of OFDM signals. Section IV analyzes the statistical distribution of PAPR of pre-equalized BHT-OFDM systems and proposes a generic simple closed-form PAPR expression. Section V gives the numerical studies and simulation results. Finally, Section VI contains the conclusion.

\section{SySTEM MODEL}

The pre-equalized BHT-OFDM works in a finite field [3], whose block diagram is shown in Fig. 1, supported by a time division duplex (TDD) with channel state information (CSI) feedback. BHT-OFDM systems offer inherent error control capability, lower PAPR and complexity, with cost of quantization noise introduced by conversion between complex field and finite field. Compared to the conventional OFDM, the key differences with the pre-equalized BHT-OFDM are:

(1) Modulation is replaced by finite-field mapping so that binary information is mapped to an element in finite-field instead of complex-field constellation point. 


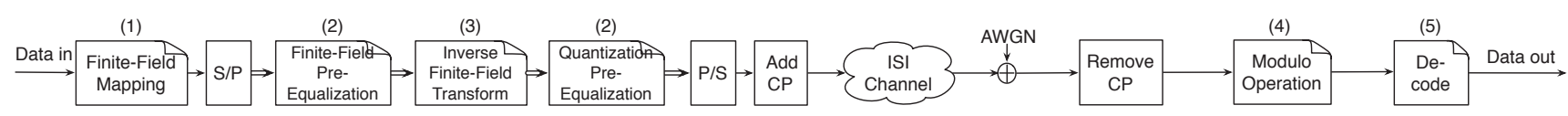

(a). Pre-Equalized BHT-OFDM system

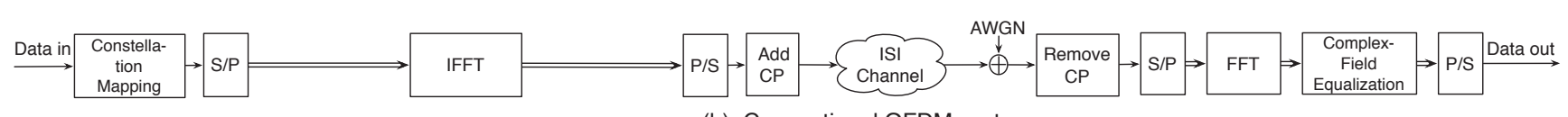

(b). Conventional OFDM system

S/P: Serial to Parallel Convert $\quad$ P/S: Parallel to Serial Convert $\quad$ CP: Cyclic Prefix

Fig. 1. Comparison of a pre-equalized BHT-OFDM and conventional OFDM system.

(2) Finite-field equalization replaces the conventional complex field equalization to eliminate the inter-symbol interference (ISI). Here, we shift the finite field equalization to the transmitter as a finite field pre-equalization. The quantization pre-equalization quantizes the original channel to some finite integer coefficients.

(3) The IFFT is replaced by an inverse finite-field transform, which is specified as IBHT in this paper.

(4) A new signal conversion module (modulo operation) is used at the receiver front-end to convert the received signal to an element in finite field.

(5) There is no FFT at receiver. Instead, a decoding module is utilized to exploit the inherent error correction capability of the received signal.

Now, we follow [3] to define BHT. A base field $\mathcal{B}$ can be extended to an extension field $\mathcal{E}_{\mathcal{B}}$, which is an $m$-dimensional vector space, and let $W_{N} \in \mathcal{E}_{\mathcal{B}}$ be an element with order $N$ where $N$ corresponds the transform length. There exists a unique normal basis $\langle\alpha\rangle=\left\{\alpha, \alpha^{p}, \ldots, \alpha^{p^{m-1}}\right\}$, where $p$ is an odd prime, in $\mathcal{E}_{\mathcal{B}}$ such that $\operatorname{tr}\left(\alpha W_{N}\right) \in \mathcal{B}$. Here, $\operatorname{tr}(\zeta)$ is the trace function defined as $\operatorname{tr}(\zeta)=\zeta+\zeta^{p}+\ldots+\zeta^{p^{m-1}}$. Define BHT as

$$
\bar{x}_{n}=\sum_{k=0}^{N-1} x_{k} \operatorname{tr}\left(\alpha W_{N}^{k n}\right),
$$

where $\bar{x}_{n}$ denotes the signal in BHT domain. On the other hand, the inverse BHT (IBHT) is defined as

$$
x_{n}=N^{-1} \sum_{k=0}^{N-1} \bar{x}_{k} \operatorname{tr}\left(\beta W_{N}^{-k n}\right),
$$

where $\langle\beta\rangle=\left\{\beta, \beta^{p}, \ldots, \beta^{p^{m-1}}\right\}$ is the unique dual basis of $\langle\alpha\rangle$.

Similar to the discrete Fourier transform (DFT), BHT satisfies the convolution property as

$$
\bar{a}_{n}=\sum_{i, j=0}^{m-1} \operatorname{tr}\left(\alpha \beta^{p^{2 i}+p^{2 j}}\right) \bar{x}_{n}^{p^{2(m-i)}} \bar{y}_{n}^{p^{2(m-j)}}
$$

where the $\left\{\bar{x}_{n}\right\},\left\{\bar{y}_{n}\right\}$ and $\left\{\bar{a}_{n}\right\}$ are the BHT of three timedomain vectors $\left\{x_{n}\right\},\left\{y_{n}\right\}$ and $\left\{a_{n}\right\}$, respectively. Due to this convolution property, circular convolutional channel matrix can be decomposed into certain sparse matrix form, instead of a diagonal matrix in OFDM systems. However, few nonzero elements only appear on the positions within the same cyclotomic cosets of the sparse matrix, which reduces the complexity of the finite-field pre-equalization. After proper frequency-domain quantization pre-equalization at transmitter and modulo operation at receiver, the received signal can be written as

$$
\mathbf{y}=\mathbf{B}^{*} \overline{\mathbf{x}}+\mathbf{n},
$$

where $\mathbf{B}^{*}$ corresponds to the IBHT. By utilizing reserved tones of OFDM, $\overline{\mathrm{x}}$ consists of redundant bits in a finite field, which causes that the vector $\mathbf{y}$ is a codeword corrupted by an AWGN vector. Therefore, the pre-equalized BHT-OFDM has inherent error correction capability and only a decoding process is needed at receiver to recover the received codeword.

Let $a(t)$ and $b(t)$ be the real and imaginary parts of the signal, respectively. The complex baseband signal is expressible as [6]:

$$
y(t)=a(t)+j b(t)=\frac{1}{\sqrt{N}} \sum_{k=0}^{N-1} A_{k} e^{j 2 \pi\left((k-(N-1) / 2) / T_{s}\right) t}
$$

where $A_{k}$ is the signal amplitude in each subcarrier $k, j=$ $\sqrt{-1}$ and $T_{s}$ is the BHT-OFDM symbol period such that the envelope of the BHT-OFDM signal $s(t)$ normalized by the average power is given by:

$$
r(t)=\sqrt{\frac{x^{2}(t)+y^{2}(t)}{P_{\mathrm{av}}}}
$$

where $P_{\mathrm{av}}=\mathbf{E}\left(\left|A_{k}\right|^{2}\right)$. By definition, we have the crest factor $(\mathrm{CF})$ that is the square root of the PAPR expressible as:

$$
C=\sqrt{\mathrm{PAPR}}=\sqrt{\frac{\max _{0 \leqslant t \leqslant T_{s}}|y(t)|^{2}}{P_{\mathrm{av}}}}=\max _{0 \leqslant t \leqslant T_{s}} r(t) .
$$

In the following sections, we are going to investigate the PAPR performance of pre-equalized BHT-OFDM schemes in comparison to that of conventional OFDM systems, by both closed-form exact analytical expression and simulation results.

\section{Conventional Analysis of the PAPR of OFDM SIGNALS}

In conventional OFDM systems, one may assume that the transmitted symbols are independent complex Gaussian 
distributed such that their amplitude is independent Rayleigh distributed. It is well known that the cumulative distribution function (CDF) of a Rayleigh distribution is given by:

$$
F(r)=\left(1-\exp \left(-r^{2}\right)\right)^{N},
$$

where $r$ is used to denote the envelope of the OFDM signal normalized by the average power, and $N$ is the length of the complex data sequences inputted to the inverse DFT (IDFT) in an OFDM transmitter, which is also equal to the number of subcarriers used for.

Notice that the above CDF expression is given by assuming that the sampled baseband OFDM signals at the Nyquist rate, denoted by $s_{l}=s\left(l T_{s} / N\right)$, are statistically independent. Therefore, $r_{l}=\left|s_{l}\right| / \sqrt{P_{\mathrm{av}}}$ is independent and identically distributed (i.i.d.) Rayleigh random variables. The corresponding probability density function (PDF) is given by:

$$
f_{r_{l}}(r)=2 r e^{-r^{2}} \text {. }
$$

However, the assumption of independent transmitted signals may not hold for many scenarios. Therefore, the analysis of correlated signals is necessary, which is classified to empirical and theoretical expressions.

An empirical approximate of the CDF of PAPR of OFDM signals with correlated distribution was derived in [4] such that

$$
F(r)=C^{-1}\left(1-\exp \left(-r^{2}\right)\right)^{\alpha N}
$$

where an empirical parameter $\alpha$ was given by 2.8 , and $C$ is a normalization factor such that $\int F(r) d r=1$. However, this empirical approximation yields discrepancies with simulation results and cannot fit well for band-limited OFDM signals even when $N$ is large [6]. Our simulation results of pre-equalized BHT-OFDM also show that in different channel realizations, the exponent parameter $\alpha$ would be less than 1 . Note that one may consider empirical modification or fine tune so as to improve the above expression. However, generally speaking, Equation (10) lacks a very rigorous theoretical justification although it may work in some cases. In brief, the above empirical approximation is simple and nice but is not general and precise.

On the other hand, the theoretical analysis of PAPR of conventional OFDM has been extensively investigated in literature. In [6], an exact distribution of the CF (cf. (7)) was derived for correlated OFDM signals, by estimating the number of peaks through level-crossing rate analysis [5]. Provided that (i) the processes $a(t)$ and $b(t)$ are ideally bandlimited Gaussian and (ii) the peaks of the OFDM signal are statistically independent, which could be true for large $N$, the distribution of PAPR is given by[6]

$$
F(r) \approx(1-\operatorname{Pr}(\rho>r))^{0.64 N},
$$

where $\operatorname{Pr}(\rho>r)$ is the probability that an arbitrary peak $\rho$ in an OFDM symbol is above $r$. Note that the above expression of the PAPR distribution is hard to compute as the numerical evaluation of $\operatorname{Pr}(\rho>r)$ requires difficult integrals, see [6]. Besides, the assumption of statically independence of peaks may hold only for very large $N$. Approximation of Equation (11) to simple computable form for removing the computation difficulty of $\operatorname{Pr}(\rho>r)$ was thus proposed for large $N$ such that

$$
F(r) \approx \begin{cases}\left(1-\frac{r e^{-r^{2}}}{\bar{r} e^{-\bar{r}^{2}}}\right)^{\sqrt{\pi / 3} N \bar{r} e^{-\bar{r}^{2}}} & \text { for } r>\bar{r} \\ 0 & \text { otherwise }\end{cases}
$$

where $\bar{r}$ is a reference level around 1.7 to 1.8 for QPSK that is determined by simulation results. The above was observed asymptotically close to Equation (11) for large $N$; however, the right hand side of Equation (12) might diverge and yield significant approximation error in some cases (for example when $r$ approaches $\bar{r}$ ).

\section{Statistical Distribution of PAPR of PRE-EQUALIZED BHT-OFDM}

In pre-equalized systems, the transmitted complex Gaussian symbols are correlated. We observe a significant difference between the PAPR distribution of pre-equalized BHT-OFDM and that of conventional OFDM. Our simulation results in Section $\mathrm{V}$ for practical systems have also shown that the amplitude of the transmitted symbols are correlated Rayleigh and Rician distributed, for frequency deep fading and flat fading channels, respectively.

In this section, we aim to derive the analytical expression of PAPR distribution of pre-equalized OFDM and then determine its PAPR performance. In brief, we have to derive CDF, $F(r)$, with respect to a number of $N$ correlated Gaussian random variables. Tractable closed-form exact expressions without approximation that can be easily computed for performance evaluation and numerical studies would be highly favorable. Therefore, we derive the analytical expression of PAPR distribution of pre-equalized BHT-OFDM and then determine its PAPR performance, based on the result of [8]. We do not follow the approach of level-crossing analysis for estimating PAPR like [6] due to two main reasons: (i) there requires several assumptions that the result may be precise only under certain conditions, and (ii) the numerical evaluation of the analytical expression Equation (11) is difficult or may require some parameter estimations that have to be conducted in advance (see Equation (12)) and may vary from case to case.

Recall that when $r_{l}, l=1,2, \ldots, N$ are i.i.d. Rayleigh random variables (i.e., uncorrelated), it is clear that:

$$
F\left(r_{1}<r, \ldots, r_{N}<r\right)=\left(\operatorname{Pr}\left(r_{l}<r\right)\right)^{N}=\left(1-e^{-r^{2}}\right)^{N} \text {. }
$$

As the real and imaginary parts $a$ and $b$ of signals are Gaussian distributed, while they are independent, the envelope $r$ is still Rayleigh. Without loss of generality, the PDF expression in Equation (9) holds as usual. However, $r_{l}$ is not always independent, for example in the case of pre-equalized BHTOFDM, in which the transmitted signals are affected by channels through pre-equalization. Therefore, by definition we have:

$$
F_{c}(r)=\operatorname{Pr}\left(\max _{0 \leqslant l<N} r_{l}<r\right)=\operatorname{Pr}\left(r_{0}<r, \ldots, r_{N}<r\right),
$$


where $C$ refers to the crest factor (CF) that is the square root of PAPR.

For correlated Gaussian random variables, one can derive their joint probability density function by considering multivariate correlated Gaussian distributions, especially when the variables are evenly correlated or exponentially correlated [9]. However, the provided joint PDF requires a multidimensional ( $N$-fold) integration which is hard to compute (where the complexity grows exponentially), especially for large $N$.

A recent advancement of novel single integral representation for the above multivariate PDF is reported in [8]; one can see that it could greatly facilitate the performance evaluation and analysis for digital communications. Given that the transmitted symbols have zero-mean complex Gaussian distribution and the magnitude is Rayleigh distributed with unit mean square value, i.e., $\mathbf{E}\left(|\cdot|^{2}\right)=1$, we have [8]:

$$
\begin{aligned}
& F_{\text {Rayleigh }}\left(r_{1}, r_{2}, \ldots, r_{N}\right)= \\
& \int_{t=0}^{\infty} e^{-t} \prod_{k=1}^{N}\left[1-\mathcal{Q}\left(\sqrt{\frac{t \rho_{k}^{2}}{\sigma_{k}^{2}}}, \frac{r_{k}}{\sigma_{k}}\right)\right] d t,
\end{aligned}
$$

where $\mathcal{Q}(m, n)$ is the first order Marcum $\mathcal{Q}$-function and $\rho_{k}$ is the correlation coefficient by the following correlation model [11] in describing the underlying complex Gaussian random variables:

$$
G_{k}=\left(\sqrt{1-\rho_{k}^{2}} a_{k}+\rho_{k} a_{0}\right)+i\left(\sqrt{1-\rho_{k}^{2}} b_{k}+\rho_{k} b_{0}\right),
$$

with respect to the common reference $G_{0}=a_{0}+i b_{0}$, where $k=1,2, \ldots, N$. Note that $i^{2}=-1, \rho_{k} \in(-1,1) \backslash\{0\}$, $a_{k}$ and $b_{k}$ are independent and $a_{k}, b_{k} \sim N(0,1 / 2), G_{k}$ has a zero-mean complex Gaussian distribution $N_{c}(0,1)$ and consequently $\left|G_{k}\right|$ is Rayleigh distributed with expectation $\mathbf{E}\left(\left|G_{k}\right|^{2}\right)=1$. Notice that the correlation structure in Equation (15) generates correlated Rayleigh envelopes and the crosscorrelation of $G_{k}$ and $G_{j}$ is given by $\rho_{k} \rho_{j}$ for $j \neq k$. In case of equal correlation, one can have $\rho_{k}=\rho$ for all $k$ such that

$$
G_{k}=\left(\sqrt{1-\rho^{2}} a_{k}+\rho a_{0}\right)+i\left(\sqrt{1-\rho^{2}} b_{k}+\rho b_{0}\right) .
$$

Similarly, we can also get the CDF of the correlated Rician distribution as [8]:

$$
\begin{aligned}
& F_{\text {Rician }}\left(r_{1}, r_{2}, \ldots, r_{N}\right)= \\
& \begin{aligned}
\int_{R=0}^{\infty} \exp (-t) \exp ( & \left.-\left(m_{1}^{2}+m_{2}^{2}\right)\right) I_{0}\left(2 \sqrt{t\left(m_{1}^{2}+m_{2}^{2}\right)}\right) \\
\times & \prod_{k=1}^{N}\left[1-\mathcal{Q}\left(\sqrt{\frac{t \rho_{k}^{2}}{\sigma_{k}^{2}}}, \frac{r_{k}}{\sigma_{k}}\right)\right] d t, \quad \text { (18) }
\end{aligned}
\end{aligned}
$$

where $I_{0}(\cdot)$ is the zero-th order modified Bessel function of the first kind, and random variables $a_{0} \sim N\left(m_{1}, 1 / 2\right), b_{0} \sim$ $N\left(m_{2}, 1 / 2\right)$.

Based on Equations (15) and (18), we can easily get the complementary cumulative distribution function (CCDF) of the PAPR, which is the most frequency used PAPR performance measure.

\section{Numerical and Simulation Results}

In this section, we conduct numerical evaluation of the analytical expressions and also computer simulations to show the CDF of the pre-equalized BHT-OFDM signals, comparing with that of conventional OFDM systems. The WiMAX SUI and 3GPP WINNER Phase II Models [10] have been considered in the simulation. The transform length is chosen as $N=128$. For both numerical study and simulations, we model pre-equalized BHT-OFDM symbols and their correlation by Equation (16). For an arbitrary set $\rho_{k}$ with common components $a_{0}$ and $b_{0}$, the joint $\mathrm{CDF}$ is given by Equation (15). We compute $F\left(r_{1}, r_{2}, \ldots, r_{N}\right)$ by representing an $N$ length complex data sequence of pre-equalized BHT-OFDM as follows:

- $a_{k}, b_{k} \sim N(0,1 / 2)$, where $a_{k}$ and $b_{k}$ are independent.

- $\rho_{k}$, where $\rho_{k} \in(-1,1) \backslash\{0\}$.

- $G_{k}$ has a zero-mean complex Gaussian distribution $N_{C}(0,1)$ and $\mathbf{E}\left(\left|G_{k}\right|^{2}\right)=1$.

- $\sigma_{k}^{2}=\frac{1-\rho_{k}^{2}}{2}$, for all $k$.

Then, a CDF based on Equation (15) can be evaluated accordingly. In the numerical studies, we consider that $\rho_{k}=\rho$ for simplicity.

Based on WiMAX SUI channel model, the simulation results show that when there exists deep fading on frequency domain, the amplitude of transmitted symbols matches to the corrected Rayleigh distribution very well, as shown in Fig. 2. The corresponding PAPR in comparison with conventional OFDM is shown in Fig. 3. We can see in the deep fading channel, pre-equalized BHT-OFDM outperforms conventional OFDM, in terms of PAPR performance. It is shown that the $0.1 \%$ PAPR of the conventional OFDM signal is 10.8 $\mathrm{dB}$, and that of pre-equalized BHT-OFDM is around $8 \mathrm{~dB}$, resulting in about $2.8 \mathrm{~dB}$ reduction. We also present analytical CCDF of PAPR based on Equation (15) in Fig. 3. It shows that theoretical analysis result matches very well with the simulation result.

In the case of frequency flat fading channels, the transmitted symbols are correlated Rician distribution instead of Rayleigh distribution, as shown in Fig. 4. In this case the PAPR performance is well controlled compared with OFDM systems, the $0.1 \%$ PAPR of pre-equalized BHT-OFDM significantly reduces to $5.1 \mathrm{~dB}$, resulting in remarkable $5.65 \mathrm{~dB}$ reduction, as shown in Fig. 5. The theoretical analysis result from Equation (18) matches well with the simulation result for preequalized BHT-OFDM as well. Table I summarizes the overall PAPR reduction. One can see that pre-equalized BHT-OFDM clearly outperforms in the power efficiency.

TABLE I

PAPR REDUCTION UNDER FREQUENCY FLAT AND DEEP FADING

\begin{tabular}{l|c|c|c|c}
\hline CCDF threshold & 0.5 & $10^{-1}$ & $10^{-2}$ & $10^{-3}$ \\
\hline \hline Deep fading & $1.18 \mathrm{~dB}$ & $1.54 \mathrm{~dB}$ & $2.16 \mathrm{~dB}$ & $2.76 \mathrm{~dB}$ \\
\hline Frequency flat & $3.00 \mathrm{~dB}$ & $3.97 \mathrm{~dB}$ & $4.84 \mathrm{~dB}$ & $5.65 \mathrm{~dB}$ \\
\hline
\end{tabular}



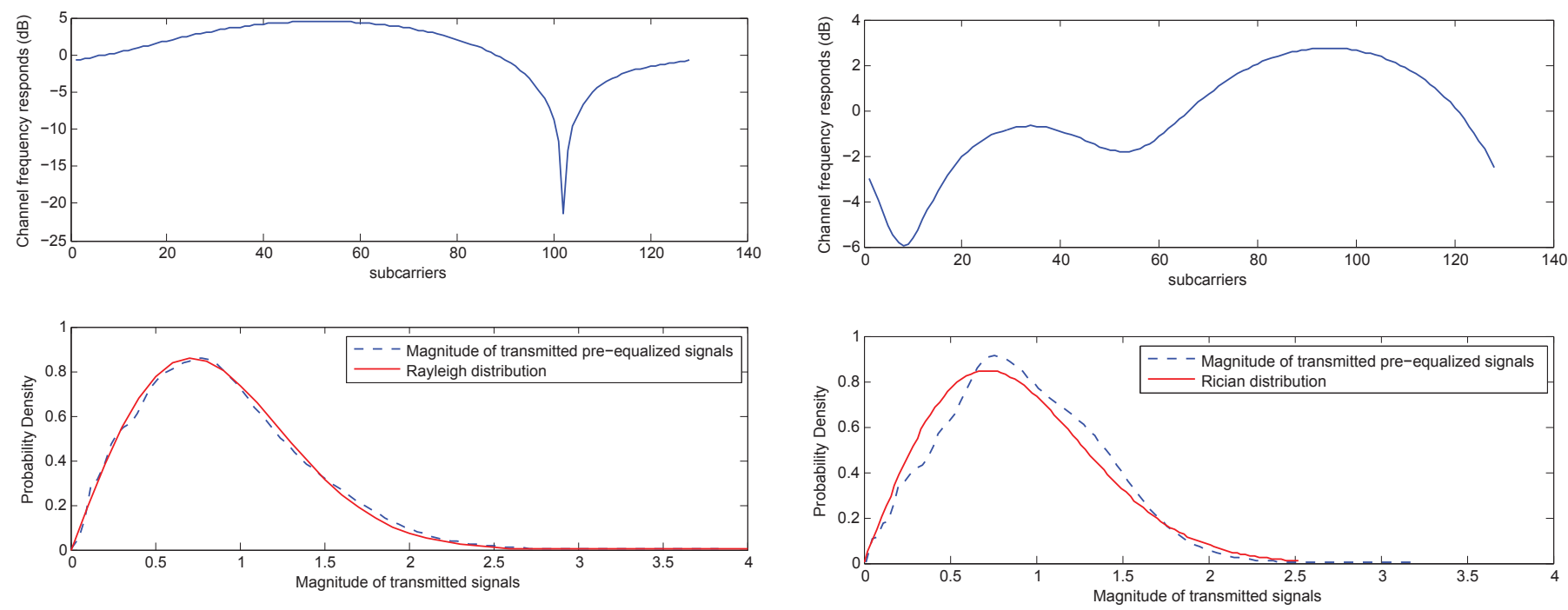

Fig. 2. Probability density of signals in a frequency deep fading WiMAX SUI channel



Fig. 4. Probability density of signals in a frequency flat fading WiMAX SUI channel

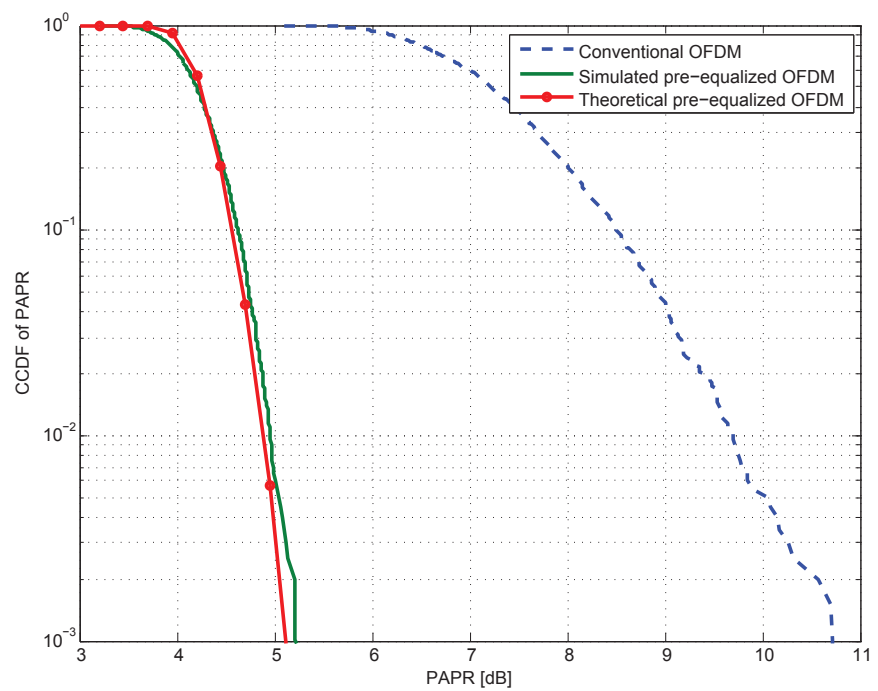

Fig. 3. Comparison of simulation results of the CCDF of the PAPR in a frequency deep fading WiMAX SUI channel

In order to justify the results, we simulate OFDM and BHTOFDM by an more experimental model, WINNER Phase II channel model, in Fig. 6 and Fig. 7. We can see BHT-OFDM outperforms OFDM by around $2 \mathrm{~dB}$ at CCDF level of $10^{-3}$. And the theoretical result matches simulation result very well.

On the other hand, most communication systems use high power amplifier (HPA) at the transmitter to obtain sufficient transmits power, therefore, HPA efficiency is a good monitor to measure the system energy efficiency. With an ideal linear model for HPA, HPA efficiency can be defined as [2]

$$
\epsilon=0.5 / \text { PAPR, }
$$

which also equals to the ratio of the average output power and the a constant DC power. In the scenario of frequency deep fading channels, at $10^{-3} \mathrm{CCDF}$ level, PAPR $=8.03$ $\mathrm{dB}(\approx 6.35)$ and $\mathrm{PAPR}=10.79 \mathrm{~dB}(\approx 12.00)$, respectively,

Fig. 5. Comparison of simulation results of the CCDF of the PAPR in a frequency flat WiMAX SUI channel

referring to Fig. 3. Therefore BHT-OFDM improves the HPA efficiency to $\epsilon=0.5 / 6.35=7.87 \%$, which is around 2 times of $4.17 \%$ of conventional OFDM. For flat fading case, at $10^{-3}$ CCDF level, BHT-OFDM improves HPA efficiency more than $300 \%$, comparing to conventional OFDM.

Therefore, correlated Rayleigh and Rician distribution accurately describe the transmitted pre-equalized BHT-OFDM signals over frequency deep fading and flat channel, respectively. And the corresponding CDF expressions measure PAPR performance very well.

\section{CONCLUSION}

We show by exact analytical expressions and simulation results that pre-equalization BHT-OFDM provides better PAPR performance than traditional OFDM systems. We develop a 

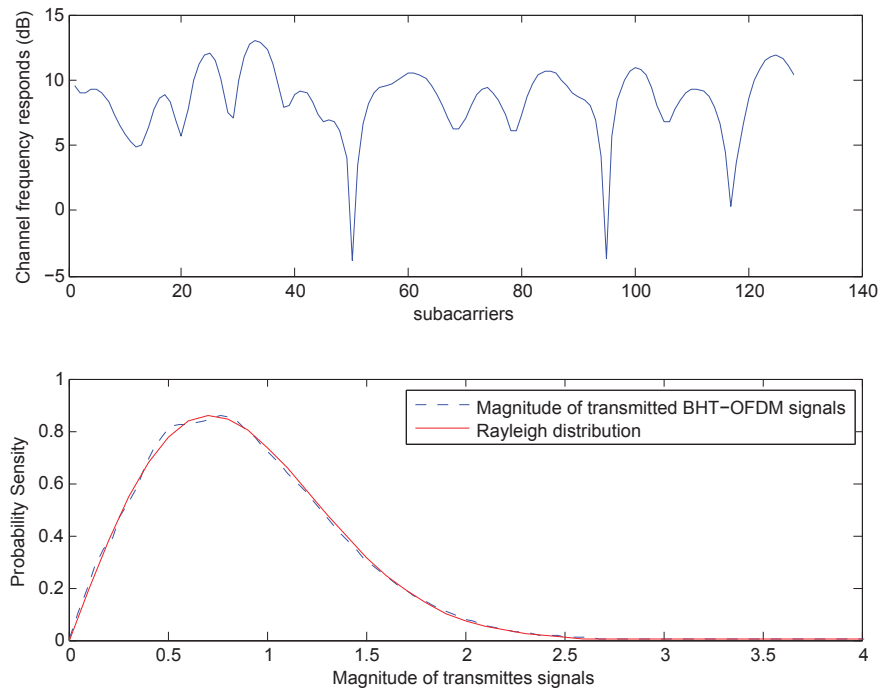

Fig. 6. Probability density of signals with WINNER Phase II channel model

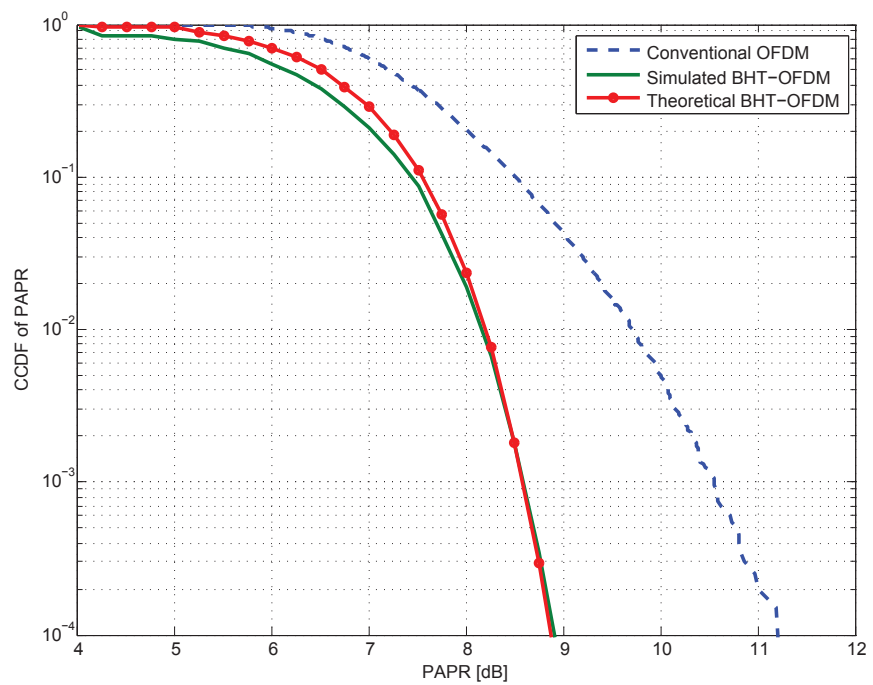

Fig. 7. Comparison of simulation results of the CCDF of the PAPR with WINNER Phase II channel model

tractable analytical closed-form expression, which is suitable for correlated Gaussian distributed signals, and verify the result with comprehensive simulations. Comparisons show that the analytical and simulation results are well matched. Our study suggests that pre-equalized BHT-OFDM is not only achieving better BER performance but also reducing PAPR significantly, and has a high potential for future green wireless communication systems for LTE-beyond or 5G. More importantly, the analytical expressions and method are suitable for a large scope of systems with correlated distributed signals.

\section{ACKNOWLEDGMENT}

The research leading to these results has received funding from the European Union Seventh Framework Programme (FP7/2007-2013) under grant agreement n. 257740 (Network of Excellence "TREND").
The work presented in this paper has been carried out at LINCS (www.lincs.fr) and ITR, University of South Australia. We would like to thank Laurent Thomas and Alberto Conte of Alcatel-Lucent Bell Labs, as well as Prof. Alex Grant at University of South Australia for their valuable discussion and support.

\section{REFERENCES}

[1] E. Dahlman, S. Parkvall and J. Skold, "4G: LTE/LTE-advanced for mobile broadband," Oxford. Academic Press, 2011.

[2] Tao Jiang and Yiyan Wu, "An overview: peak-to-average power ratio reduction techniques for OFDM signals," IEEE Trans. Broadcasting, vol. 54, no. 2, June 2008.

[3] H. Liu, L. Luo and P. Z. Fan, "A pre-equalized transmission based on basefield Hartley transform over multi-path fading channels," Proc. IEEE Vehicular Technology Conference (VTC), pp. 1-6, Sept. 2011.

[4] R. van Nee and A. de Wild, "Reducing the peak-to-average power ratio of OFDM," Proc. VTC, pp. 2072-2076, May. 1998.

[5] N. B. Mandayam, P.-C. Chen, and J. M. Holtzman, "Minimum duration outage for CDMA cellular systems: a level crossing analysis," Wireless Personal Commun., Springer, vol. 7, no. 2-3, pp. 135-146, Aug. 1998.

[6] H. Ochiai and H. Imai, "On the distribution of the peak-to-average power ratio in OFDM signals," IEEE Trans. Commun., vol. 49, no. 2, pp. 282 289, Feb. 2001

[7] 3GPP TS 36.211, "Evolved universal terrestrial radio access: physical channels and modulation (Release 10)," Tech. Spec v10.2.0, June 2011.

[8] N. C. Beaulieu and K. T. Hemachandra, "Novel simple forms for multivariate Rayleigh and Rician distributions with generalized correlation," Proc. IEEE Globecom, pp. 1-6, 2010.

[9] R. K. Mallik, "On the multivariate Rayleigh and exponential distributions," IEEE Trans. Inform. Theory, vol. 49, pp. 1499-1515, June 2003.

[10] L. Hentila, P. Kyosti, M. Kaske, M. Narandzic, and M. Alatossava. (2007, December.) MATLAB implementation of the WINNER Phase II Channel Model ver1.1 [Online]. Available: https://www.ist-winner.org/ phase_2_model.html.

[11] Y. Tong, "The multivariate Normal Distribution," New York: SpringerVerlag, 1990 